\title{
A CLASS OF MEROMORPHIC FUNCTIONS WITH NO WANDERING DOMAINS
}

\author{
Gwyneth M. Stallard
}

\begin{abstract}
We consider the class of meromorphic functions with finitely many fixed points, that is the class of functions of the form $f(z)=z+R(z) e^{g(z)}$, where $R$ is rational and $g$ is entire. We show that, in the case where $g$ is a polynomial, such a function has certain properties which allow us to apply the method of proof of [2, Theorem 6.1] to show that its set of normality can have no wandering domains.
\end{abstract}

\section{Introduction}

In the following we will let $f: \mathbf{C} \rightarrow \hat{\mathbf{C}}$ denote a non-linear meromorphic function and $f^{n}, n \in \mathbf{N}$, the $n$-th iterate of $f$. The set of normality, $N(f)$, is defined to be the set of points $z \in \mathbf{C}$ such that the sequence $\left(f^{n}\right)$ is defined, meromorphic and forms a normal family in some neighbourhood of $z$. It is easy to see that $N(f)$ is open and has the property of complete invariance under $f$, that is $z \in N(f)$ if and only if $f(z) \in N(f)$. The complement $J(f)$ of $N(f)$ is called the Julia set. This set is clearly closed and contains all the poles of $f$ and their pre-images. More details of these and other basic properties of the sets $N(f)$ and $J(f)$ can be found in [8] and [9] for entire functions and in [3] for meromorphic functions with at least one pole.

For any meromorphic function $f$ it can easily be seen that a component $U$ of the set of normality, $N(f)$, must be mapped by $f$ into a component $V$ of $N(f)$. If $f^{n+m}(U) \cap f^{n}(U) \neq \emptyset$ for some integers $n \geq 0, m>0$ then we say that $f^{n}(U)$ is a periodic domain and, if this fails to hold for $n=0, U$ is a pre-periodic domain. If, however, $f^{n}(U) \cap f^{m}(U)=\emptyset$ for all integers $n, m \geq 0, n \neq m$, then $U$ is called a wandering domain of $f$. The situation where $U$ is periodic is well understood, and such domains can arise in only a small number of ways. In the case where $f$ is a rational function Sullivan $[13,14]$ proved the following important result thus solving a problem which had been open since the time of Fatou and Julia.

Theorem A. A rational function whose degree is at least 2 has no wandering domains.

1991 Mathematics Subject Classification: 30D05. 
This result cannot, however, be extended to cover all meromorphic functions. Indeed several examples of transcendental entire functions with wandering domains are known. One such example is the function $f(z)=z-1+2 \pi i+e^{-z}$ quoted by Sullivan in [13]. It is, however, possible to extend the result to certain classes of meromorphic functions.

One such class is the class of transcendental functions whose inverse functions have only finitely many singularities as proved by Eremenko and Lyubich in [7], and by Goldberg and Keen in [10]. Goldberg and Keen obtain their result by a method which is based on Bers' version [6] of Sullivan's proof of Theorem A. This proof, however, involves the theory of Teichmüller spaces. A similar result has been obtained by Baker [2, Theorem 6.1]. His method of proof avoids the use of Teichmüller spaces by constructing an explicit family of quasiconformal maps. The precise form of his theorem is as follows.

Theorem B. Suppose that the transcendental entire function $f$ has the following properties:

(i) there are finitely many singularities of $f^{-1}$;

(ii) the components of $N(f)$ are simply connected;

(iii) there is a constant $K>1$ and a finite set of real parameters $X_{1}, \ldots, X_{n(K, f)}$ such that every entire function of the form $f_{\Phi}=\Phi f \Phi^{-1}$, where $\Phi$ is $K$ quasiconformal, and $\Phi$ fixes $0,1, \infty$, can be expressed uniquely in terms of $X_{1}, \ldots, X_{n(K, f)}$

(iv) if in (iii) $\Phi=\Phi(t, z)$ depends on a system of parameters $t$ which vary in an open neighbourhood $\mathcal{I}$ of the origin of $\mathbf{R}^{M}$, for some $M$, in such a way that $\Phi(0, z)=z$ and $\Phi(t, z) \in C^{1}(\mathcal{I})$ for fixed $z$, then in some open subset of $\mathcal{I}$ the parameters $X_{i}$ are also $C^{1}$-functions of $t$.

Then $f$ has no wandering domains.

In a recent series of papers Baker, Kotus and Lü Yinian have proved several results concerning the iteration of meromorphic functions. In [5] they show that the results on wandering domains can be extended to cover the class of meromorphic functions which are not rational functions of degree less than 2 and whose inverse functions have only finitely many singularities.

By examining the conditions of Theorem B and its proof and the recent work on meromorphic functions we are led to consider meromorphic functions with finitely many fixed points. Such functions are of the form $f(z)=z+R(z) e^{g(z)}$ where $R$ is a rational function and $g$ is entire. We are able to prove the following result.

Theorem C. $A$ function $f$ of the form $f(z)=z+R(z) e^{Q(z)}$, where $R$ is a rational function, $Q$ is a polynomial, and $f$ is not a rational function of degree less than 2 , has no wandering domains.

The class of functions in Theorem $\mathrm{C}$ with $Q$ constant is simply the class of rational functions of degree at least 2 for which the result is already known. The 
remaining work is devoted to the proof of Theorem $\mathrm{C}$ for the class of functions where $Q$ is not a constant. We denote this class by $F$.

We claim that if $f$ is a function in $F$ that is not entire then the set

$$
O^{-}(\infty)=\left\{z: f^{n}(z)=\infty \text { for some } n \in \mathbf{N}\right\}
$$

contains infinitely many points and hence, from [3, Lemma 1], $J(f)=O^{-}(\infty)^{\prime}$. For suppose that there exists a function $g$ in $F$ which is not entire and for which the set $O^{-}(\infty)$ is finite. It follows from Picard's theorem that $g$ must be of the form

$$
g(z)=\alpha+(z-\alpha)^{-k} e^{h(z)}
$$

where $h$ is an entire function and $k$ is a positive integer. Thus $g(z)=\infty$ only at $z=\alpha$ and $g(z)=\alpha$ has no roots. As $g$ is in the class $F$, it also follows that $g(z)=z$ has only finitely many roots. Thus the function

$$
G(z)=\frac{g(z)-z}{\alpha-z}
$$

takes each of the values $0,1, \infty$ at only finitely many points. It then follows by Picard's theorem that $G$ and hence $g$ are rational functions, thus giving a contradiction.

We also note that, for any function $f$ in $F, f^{n}$ is analytic in $N(f)$ for each $n \in \mathbf{N}$.

We will show that a slightly adapted form of the proof of Theorem B can be used to prove Theorem $\mathrm{C}$. We first consider the effect of allowing $f$ to be a meromorphic function instead of just entire. The proof of Theorem B uses the fact that, if $f$ is entire, the fixed points of $f$ are dense in $J(f)$. It was shown in [3, Theorem 1] that if $f$ is meromorphic and $O^{-}(\infty)$ is infinite then the fixed points of $f$ are dense in $J(f)$. As $O^{-}(\infty)$ is infinite for all functions in the class $F$, the argument still holds. The rest of the proof follows through easily for meromorphic functions satisfying conditions (i) to (iv) with 'entire' replaced by 'meromorphic' in condition (iii) and in a few places in the proof. In the following work we consider how each of the four conditions of Theorem B are used in the proof and show that functions in the class $F$ satisfy similar conditions which are sufficient to allow this method of proof to be used.

\section{Singularities of $f^{-1}$}

We first consider the condition (i) of Theorem B and note that this is needed only to ensure that if $f$ has a wandering domain $U$ then $f^{n}(U)$ contains no singularities of $f^{-1}$, for large $n \in \mathbf{N}$. We show that this is in fact the case for all functions in the class $F$. The finite singularities of $f^{-1}$ arise in one of two ways. Firstly, suppose that $z \in \mathbf{C}$ and $f^{\prime}(z)=0$. Then $z$ is defined to be a critical 
point of $f$ and its image $f(z)$ to be a critical value of $f$. The critical values of $f$ are clearly singularities of $f^{-1}$. The other type of singularity is known as a transcendental singularity. A point $\alpha$ is said to be a transcendental singularity of $f^{-1}$ if there is a curve $\Gamma$ in $\mathbf{C}$ such that, as $z \rightarrow \infty$ on $\Gamma, f(z) \rightarrow \alpha$ on $f(\Gamma)$. For a function in the class $F$ we first show that there are no problems due to its critical values by showing that all but finitely many of its critical points are contained in invariant domains of $f$ and hence in invariant components of $N(f)$.

Theorem 2.1. If $f$ is in the class $F$ then all but finitely many of its critical points are contained in invariant domains.

Proof. We first observe that $f$ has an infinite number of critical points. It follows from Nevanlinna's theory of meromorphic functions (see, for example, [11, Theorem 3.3], that if $g$ is a transcendental meromorphic function and if $g(z)=0$ has only finitely many solutions then $g^{\prime}(z)=c$, where $c$ is a constant, $c \neq 0$, has infinitely many solutions. We apply this to the function $f(z)-z$ and deduce that $f^{\prime}(z)=0$ has an infinite number of solutions.

As the result we require is invariant under conjugation we may write $f(z)=$ $z+\lambda S(z) e^{Q(z)} / T(z)$ where $\lambda$ is a non-zero constant and $S, T, Q$ are polynomials having leading coefficients equal to 1 . We write $R(z)=S(z) / T(z),(\operatorname{deg} S-$ $\operatorname{deg} T)=n$ and $\operatorname{deg} Q=m$. Differentiating, we see that

$$
f^{\prime}(z)=1+\lambda\left[R^{\prime}(z)+R(z) Q^{\prime}(z)\right] e^{Q(z)} .
$$

For the sake of simplicity we will write $U(z)=R^{\prime}(z)+R(z) Q^{\prime}(z)$.

We now put $t=Q(z)$. In a neighbourhood of $\infty$ the branches of the inverse function $z=Q^{-1}(t)$ have an expansion of the form

$$
z=t^{1 / m}+c_{0}+c_{1} t^{-1 / m}+\cdots
$$

where $c_{0}, c_{1}$ are constants. So $Q^{-1}(t)$ has $m$ branches in the region $A=\{t$ : $\left.|t|>M, t \notin \mathbf{R}^{+}\right\}$, where $M$ is a sufficiently large constant. A particular branch $Q_{j}^{-1}(t)$ is defined by taking $\arg t^{1 / m}$ in the interval $(2 \pi(j-1) / m, 2 \pi j / m)$ where $j$ is an integer, $1 \leq j \leq m$. For sufficiently large $M, Q_{j}^{-1}$ maps $A$ univalently onto a region $B_{j}$.

From (2.1) it is clear that the critical points, $z_{c}$, of $f$ satisfy

$$
\lambda U\left(z_{c}\right) e^{Q\left(z_{c}\right)}=-1 .
$$

Putting $m+n-1=k$ we see that, as $|z| \rightarrow \infty$,

$$
U(z)=m z^{k}[1+O(1 / z)]
$$

and so taking the logarithm of the modulus of both sides of (2.3) gives

$$
\operatorname{Re} Q\left(z_{c}\right)=-\log \left|\lambda m z_{c}^{k}\right|+O\left(1 /\left|z_{c}\right|\right)
$$


as $\left|z_{c}\right| \rightarrow \infty$. Putting $t_{c}=Q\left(z_{c}\right)$, and noting that

$$
|Q(z)|=\left|z^{m}\right|(1+O(1 /|z|))
$$

as $z \rightarrow \infty$, it easily follows that $\left|\operatorname{Im} t_{c}\right| \rightarrow \infty$ as $\left|z_{c}\right| \rightarrow \infty$. Hence, for $\left|z_{c}\right|$ sufficiently large, $z_{c}$ is one of the values $Q_{j}^{-1}\left(t_{c}\right)$ and lies inside some $B_{j}$.

We now consider all such $z_{c}$ in one particular region $B_{j}$. From now on $t^{1 / m}$ refers to the $j$-th branch. From (2.2) and (2.4) we see that

$$
\operatorname{Re} t_{c}=-\log \left|\lambda m t_{c}^{k / m}\right|+O\left(1 /\left|t_{c}\right|^{1 / m}\right)
$$

as $\left|t_{c}\right| \rightarrow \infty$. So for any $M_{0}>M, \alpha>0$, all such $t_{c}$, with $\left|t_{c}\right|$ sufficiently large, lie in the region $X$ where

$$
X=\left\{t:|t|>M_{0}, \operatorname{Re} t<\alpha-\log \left(|\lambda| m|t|^{k / m}\right)\right\} .
$$

Now $\left.f\right|_{B_{j}}$ is conjugate to the function $F_{j}=\left.Q f Q_{j}^{-1}\right|_{A}$. To prove our theorem it is sufficient to show that all the values $t_{c}$ lie in invariant domains of $F_{j}$ for $\left|t_{c}\right|$ sufficiently large. We see that

$$
F_{j}(t)=Q\left\{Q_{j}^{-1}(t)+\lambda R\left(Q_{j}^{-1}(t)\right) e^{t}\right\}=Q\left\{Q_{j}^{-1}(t)+\lambda e^{t} t^{n / m}\left[1+O\left(t^{-1 / m}\right)\right]\right\}
$$

as $|t| \rightarrow \infty$. In $X$ we have

$$
\begin{aligned}
\left|\lambda e^{t} t^{n / m}\left[1+O\left(t^{-1 / m}\right)\right]\right| & \leq \frac{|\lambda| e^{\alpha}}{|\lambda| m}|t|^{(n-k) / m}\left[1+O\left(|t|^{-1 / m}\right)\right] \\
& =\frac{e^{\alpha}}{m}|t|^{(1-m) / m}\left[1+O\left(|t|^{-1 / m}\right)\right]
\end{aligned}
$$

as $|t| \rightarrow \infty$. Recalling that $Q_{j}^{-1}(t)=t^{1 / m}\left[1+O\left(t^{-1 / m}\right)\right]$ as $|t| \rightarrow \infty$, we deduce from (2.5) and (2.6) that, for $t \in X$,

$$
F_{j}(t)=t+\lambda m t^{(m-1+n) / m} e^{t}\left[1+O\left(t^{-1 / m}\right)\right]=t+\lambda m t^{k / m} e^{t}\left[1+O\left(t^{-1 / m}\right)\right]
$$

as $|t| \rightarrow \infty$. We also have from (2.6) that

$$
\left|F_{j}(t)-t\right| \leq e^{\alpha}\left[1+O\left(|t|^{-1 / m}\right)\right]
$$

as $|t| \rightarrow \infty$. Hence, given an $\varepsilon>0$, we choose $\alpha, M_{0}$ to ensure that

$$
\left|F_{j}(t)-t\right|<1+\varepsilon
$$

for all $t$ in $X$. 
We now put $H_{j}(t)=\lambda m t^{k / m} e^{t}$ and observe that

$$
\arg H_{j}(t)=C_{j}+\operatorname{Im} t+\frac{k}{m} \arg t, \quad 0<\arg t<2 \pi,
$$

where $C_{j}$ is a constant, gives a continuous definition of $\arg H_{j}(t)$ for $t$ in $A$. We consider the curves $\Gamma_{K}$ in $X$ on which $\arg H_{j}(t)$ is constant. Writing $t=r e^{i \theta}$ we have

$$
\Gamma_{K}=\left\{t \in X: r \sin \theta+\frac{k}{m} \theta=K-C_{j}\right\}
$$

for some constant $K$. Writing $t=x+i y$ this becomes

$$
\Gamma_{K}=\left\{t \in X: y+\frac{k}{m} \tan ^{-1}(y / x)=K-C_{j}\right\} .
$$

It follows from (2.9) and (2.10) that there exists $R>0$ such that

$$
K>R \Rightarrow \Gamma_{K} \subset\{z: \operatorname{Im} z>0\} \text { and } K<-R \Rightarrow \Gamma_{K} \subset\{z: \operatorname{Im} z<0\} .
$$

We find that on a curve $\Gamma_{K}$

$$
\frac{d y}{d x}=\frac{(k / m) y}{x^{2}+y^{2}+(k / m) x} .
$$

If $k=0$ then it is clear that $d y / d x=0$. If $k \neq 0$ then by choosing $\alpha, M_{0}$ appropriately we can ensure that

$$
\left|\frac{d y}{d x}\right|<\frac{|2(k / m) y|}{x^{2}+y^{2}}<\frac{2|k|}{|t|}<\frac{2|k|}{M_{0}}<1 / 100 .
$$

We now consider the vertical separation of two curves $\Gamma_{K}, \Gamma_{K^{\prime \prime}}$, in $X$ where $\delta K=K-K^{\prime}>0$, and either $K, K^{\prime}>R$ or $K, K^{\prime}<-R$. Take points $t, t^{\prime}$ lying on $\Gamma_{K}, \Gamma_{K^{\prime}}$ respectively such that the real part of each point is equal to $x$. Then from (2.9), (2.10) and (2.11) the vertical separation of the two points is given by

$$
\delta y+(k / m) \delta \theta=\delta K, \quad \text { where }|\delta \theta|<\pi / 2 .
$$

If we consider the case when $K, K^{\prime}>R$ and $\operatorname{Re} t>0$ then, putting $L=$ $\min \left(|t|,\left|t^{\prime}\right|\right)$, we see from Figure 2.1 that

$$
|\delta \theta|<\frac{\pi|\sin \delta \theta|}{2}<\frac{\pi S}{2 L}<\frac{\pi|\delta y|}{2 L} .
$$




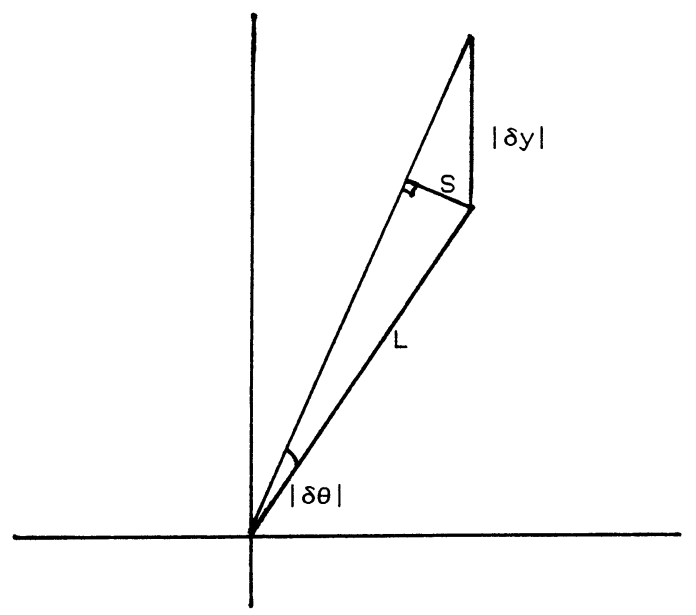

Figure 2.1.

By following the same line of argument we see that the above also holds if Ret $\leq 0$ and, similarly, if $K, K^{\prime}<-R$. Thus, by choosing $M_{0}$ and hence $L$ sufficiently large, it follows that $\delta y>0$ and indeed

$$
\delta y \pi / 3>\delta y\left(1+\frac{\pi|k|}{2 m L}\right)>\delta K>0 .
$$

We now consider the points $t_{c}$, and recall that they satisfy $\lambda U\left(Q_{j}^{-1}\left(t_{c}\right)\right) e^{t_{c}}=-1$ (see (2.3)), where $U\left(Q_{j}^{-1}(t)\right)=m t^{k / m}\left[1+O\left(t^{-1 / m}\right)\right]$ as $|t| \rightarrow \infty$. By definition, $H_{j}(t)=\lambda m t^{k / m} e^{t}$ and so

$$
H_{j}\left(t_{c}\right)=-1+O\left(t_{c}^{-1 / m}\right)
$$

as $\left|t_{c}\right| \rightarrow \infty$. Hence, for large $\left|t_{c}\right|, \arg H_{j}\left(t_{c}\right)$, defined as in (2.8), lies in an interval $((2 r+1) \pi-\pi / 6,(2 r+1) \pi+\pi / 6)$ for some integer $r\left(t_{c}\right)$. As $t_{c}$ lies on the curve $\Gamma_{K}$ where $K=\arg H_{j}\left(t_{c}\right)$ it follows that $t_{c}$ lies in the domain $V_{r}$ in $X$ bounded by the curves $\Gamma_{K_{1}}, \Gamma_{K_{2}}$ where $K_{1}=(2 r+1) \pi+\pi / 3, K_{2}=(2 r+1) \pi-\pi / 3$. For large $r$ it is clear that $V_{r}$ is contained in $A$ as $M_{0}>M$.

From previous arguments we see that for a suitable choice of $\alpha, M_{0}$ we have

i) $\left|F_{j}(t)-t\right|<3 / 2, t \in X,($ see $(2.7))$,

ii) $\arg \left(F_{j}(t)-t\right) \in(K-\pi / 100, K+\pi / 100), t \in \Gamma_{K}$, (as $\arg H_{j}(t)=K$ on $\Gamma_{K}$ and $\left.F_{j}(t)-t=H_{j}(t)\left[1+O\left(t^{-1 / m}\right)\right]\right)$,

iii) $|d y| /|d x|<1 / 100$ on each curve $\Gamma_{K}$ in $X$ (see (2.12)),

iv) $\delta y>(3 / \pi) \delta K$ for large $K$ (see (2.13) for details).

Using these results it is not difficult to see that, for large $r, F_{j}\left(V_{r}\right) \subset V_{r}$. The case where $r>0, k<0$ is illustrated in Figure 2.2. 


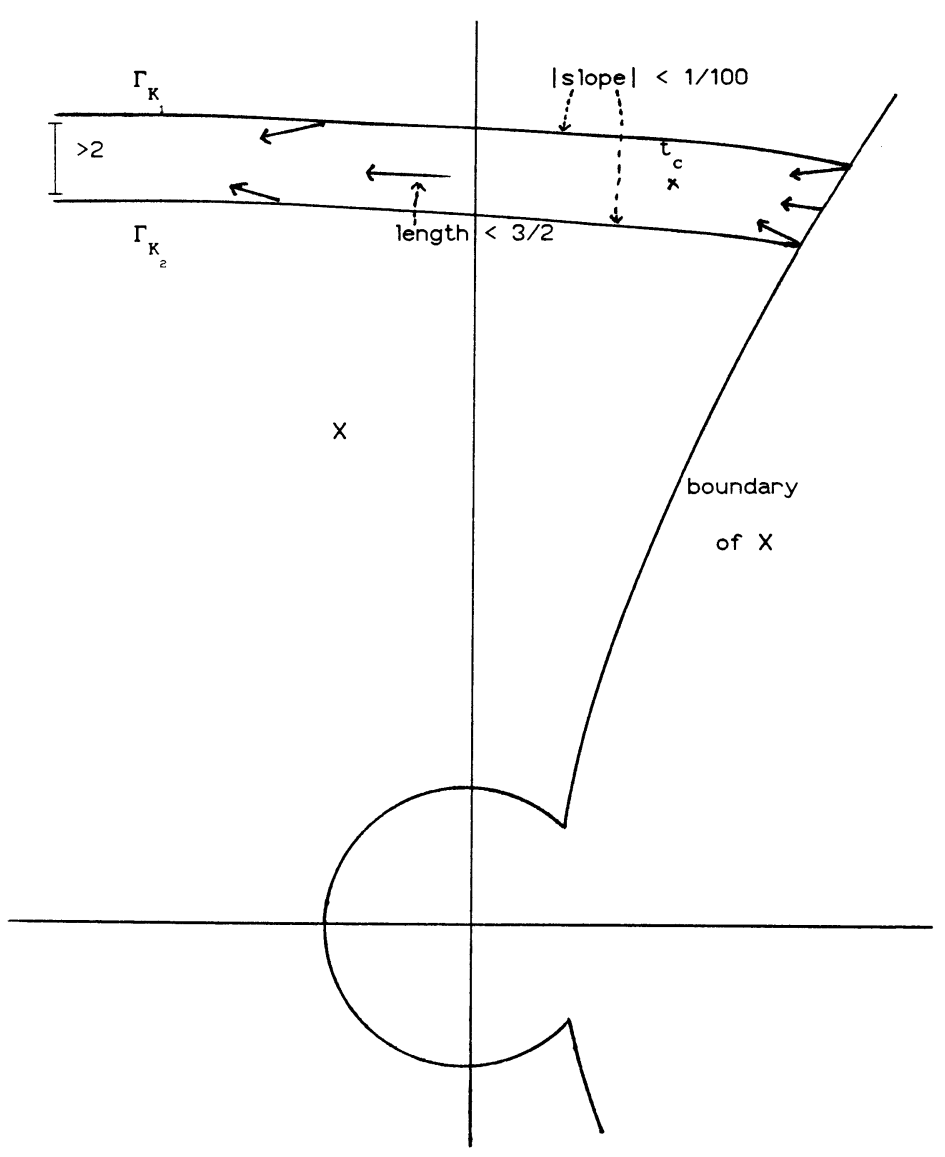

Figure 2.2.

We have shown that, for $\left|z_{c}\right|$ sufficiently large, there is a branch $Q_{j}^{-1}$ of $Q^{-1}$ such that $t_{c}=Q\left(z_{c}\right)$ lies in an invariant domain, $V_{r}$, of $F_{j}=Q f Q_{j}^{-1}$ and $Q_{j}^{-1}\left(t_{c}\right)=z_{c} \cdot Q_{j}^{-1}$ is well defined in $V_{r}$ and so $Q_{j}^{-1}\left(V_{r}\right)$ is an invariant domain of $f$ containing $z_{c}$ as required.

For any function $f$ in $F$ we now consider the transcendental singularities of $f^{-1}$. As in Theorem 2.1 we may assume that $f$ is of the form $f(z)=z+\lambda R(z) e^{Q(z)}$ where $\lambda$ is a constant, $R$ is a rational function, $Q$ is a polynomial and $R(z)=$ $z^{n}[1+o(1)], Q(z)=z^{m}[1+o(1)]$ as $z \rightarrow \infty$ for some $m, n \in \mathbf{Z}, m>0$.

We now suppose that $f^{-1}$ has a transcendental singularity at $\alpha \in \mathbf{C}$. Then by definition there is a path $\Gamma$ in $\mathbf{C}$ such that, as $z \rightarrow \infty$ on $\Gamma, f(z) \rightarrow \alpha$ on $f(\Gamma)$. So, writing $z=r e^{i \theta}$, as $z \rightarrow \infty$ on $\Gamma$ we have

$$
\begin{gathered}
\lambda R(z) e^{Q(z)}=\alpha-z+o(1)=-z[1+o(1)], \\
\left|\lambda R(z) e^{Q(z)}\right|=|\lambda| r^{n} \exp \left\{r^{m}[\cos (m \theta)+o(1)]\right\} .
\end{gathered}
$$


For $f$ to satisfy both (2.14) and (2.15) it is clear that as $z \rightarrow \infty$ on $\Gamma$ we must have $\cos (m \theta) \rightarrow 0$ and hence $\theta \rightarrow \theta_{0}$ where $\sin \left(m \theta_{0}\right)= \pm 1$.

We can also see that as $z \rightarrow \infty$ on $\Gamma$

$$
\arg \lambda R(z) e^{Q(z)}=r^{m}\left[\sin \left(m \theta_{0}\right)+o(1)\right]=r^{m}[ \pm 1+o(1)] \bmod 2 \pi .
$$

It follows from (2.14), however, that as $z \rightarrow \infty$ on $\Gamma$

$$
\arg \lambda R(z) e^{Q(z)}=\theta_{0} \pm \pi+o(1)
$$

This is clearly a contradiction, as $\arg \lambda R(z) e^{Q(z)}$ varies continuously with $z$, and so there are no finite transcendental singularities of $f^{-1}$.

It now easily follows from Theorem 2.1 that, given a function $f$ in the class $F$ and a wandering domain $U$ of $f$, the component $U_{n}$ of $N(f)$ containing $f^{n}(U)$ contains no singularities of $f^{-1}$ for large values of $n \in \mathbf{N}$.

\section{Multiply-connected domains of normality}

We now consider condition (ii) of Theorem B. The proof of Theorem B requires only that if a function $f$ has a multiply-connected wandering domain $U$ then the component $U_{n}$ of $N(f)$ which contains $f^{n}(U)$ is simply-connected for large $n \in \mathbf{N}$ which, for entire functions, is equivalent to condition (ii). In this section we show that if $f$ is a function in the class $F$ then it has no wandering domain $U$ such that $U_{n}$ is multiply-connected for all $n \in \mathbf{N}$. In order to prove this we first consider the following result proved by Baker [2, Theorem 3.1].

Lemma 3.1. If $f$ is transcendental entire and $U$ is a multiply-connected component of $N(f)$ then $U$ is a wandering domain, and every component of $N(f)$ is bounded. Further, $f^{n} \rightarrow \infty$ in $U$ as $n \rightarrow \infty$ and $U$ contains a Jordan curve $\gamma$ such that, for large $n$, the curve $\gamma_{n}=f^{n}(\gamma)$ in $U_{n}$ is at a large distance from 0 and has a non-zero winding number about 0.

We see from this that if $f$ is an entire function in the class $F$ and if $U$ is a multiply-connected wandering domain of $f$ then $U$ contains a curve $\gamma$ whose iterates nest around $\infty$. We will show that all functions in $F$ have a similar property. To do this we need the following result concerning meromorphic functions proved in [4, Lemma 2.1].

Lemma 3.2. Suppose that $f$ is a meromorphic function such that $O^{-}(\infty)$ contains infinitely many points and that $\left(f^{n}\right)$ contains a subsequence with a nonconstant limit function in a component $N_{0}$ of $N(f)$. Then $N_{0}$ is either periodic or pre-periodic.

We are now able to show that a weaker form of Lemma 3.1 is true for all functions in the class $F$. 
Lemma 3.3. Suppose that $f$ is a function in the class $F$ and that $U$ is a wandering domain of $f$ such that $U_{n}$ is multiply-connected for all $n \in \mathbf{N}$. Then, for some $k \in \mathbf{N}, U_{k}$ is bounded and there exists a Jordan curve $\gamma$ in $U_{k}$ such that for any $M>0, M \in \mathbf{R}$, there exists $L(M) \in \mathbf{N}$ and a continuum $\delta_{L(M)} \subset$ $\gamma_{L(M)}=f^{L(M)}(\gamma)$ such that $\delta_{L(M)} \subset\{z:|z|>M\}$, with one of the components of the complement of $\delta_{L(M)}$ being bounded and containing $\{z:|z|<M\}$.

Proof. As already observed, if $f$ is entire then the result follows immediately from Lemma 3.1. Now suppose that $f$ is a function in the class $F$ with poles $p_{1}, \ldots, p_{m}$ and that $U$ is a wandering domain of $f$ such that $U_{n}$ is multiplyconnected for all $n \in \mathbf{N}$. From Section 2 we know that there exists $k \in \mathbf{N}$ such that, putting $V=U_{k}$, each $V_{n}$ contains no singularities of $f^{-1}$.

We take a Jordan curve $\gamma(t)$ in $V, \gamma:[0,1] \rightarrow V, \gamma(0)=p$, which is not null-homotopic in $V$. We claim that, for each $n \in \mathbf{N}, \gamma_{n}$ is not null-homotopic in $V_{n}$. For suppose not then, for some $m \in \mathbf{N}, \gamma_{m} \sim f^{m}(p)$ in $V_{m}$ where $\sigma \sim \psi$ if and only if $\sigma$ is homotopic to $\psi$. We now take the branch $g$ of $f^{-m}$ such that $g f^{m}(p)=p$. As there are no singularities of $f^{-1}$ in each $V_{n}, 1 \leq n \leq m$, it follows that the continuation of $g$ around $\gamma_{m}$ gives $\gamma$. Hence $g$ can be used to lift the homotopy $\gamma_{m} \sim f^{m}(p)$ in $V_{m}$ to the homotopy $\gamma \sim p$ in $V$. This is clearly a contradiction.

We now take a Jordan curve $\gamma$ in $V$ such that, for each $r \in \mathbf{N}, \gamma_{r}$ is not null-homotopic in $V_{r}$. It is clear that, for each $r$, one of the bounded components of $\mathbf{C} \backslash \gamma_{r}$, say $D_{r}$, must contain a point in $J(f)$ and hence, as $J(f)=O^{-}(\infty)^{\prime}$, a point in $O^{-}(\infty)$. We denote by $n(r)$ the least non-negative integer such that $f^{n(r)}$ maps a point in $D_{r}$ to one of the poles $p_{i(r)}$ of $f$, where $i(r) \in\{1, \ldots, m\}$. As $f^{n(r)}$ is analytic in $D_{r}$ it follows that $f^{n(r)}\left(D_{r}\right)$ is a bounded domain containing $p_{i(r)}$. Without loss of generality we may assume that $i(r)=1$ for infinitely many values of $r$.

From Lemma 3.2 we see that all convergent subsequences of $\left(f^{r+n(r)}\right)$ in $V$ must have constant limits and so the spherical diameter of $\gamma_{r+n(r)}$ must tend to zero as $r \rightarrow \infty$. So there must either be a subsequence of $\left(\gamma_{r+n(r)}\right)$ which tends to $\infty$ or a subsequence which tends to $p_{1}$.

We now take $M$ to be a real value such that $M>\max \left(\left|p_{1}\right|, \ldots,\left|p_{m}\right|\right)$. In the case where there is a sequence of curves $\dot{\gamma}_{r(k)+n(r)}$ tending to $\infty$, there exists $r(k)$ such that $\gamma_{r(k)+n(r)} \subset\{z:|z|>M\}$. As $p_{1} \in f^{n(r)}\left(D_{r(k)}\right)$, it follows that $f^{n(r)}\left(D_{r(k)}\right) \supset\{z:|z|>M\}$. In this case we take $\delta_{L(M)}=\partial f^{n(r)}\left(D_{r(k)}\right) \subset$ $\gamma_{r(k)+n(r)}$.

In the case where there is a sequence of curves $\gamma_{r(k)+n(r)}$ tending to $p_{1}$, as $p_{1}$ is a pole of $f$, it follows that there exists $r(k)$ such that $\gamma_{r(k)+n(r)+1} \subset\{z$ : $|z|>M\}$, and also that $f^{n(r)+1}\left(D_{r(k)}\right)$ is a full neighbourhood of $\infty$ contained in $\{z:|z|>M\}$. So we put $L(M)=n(r)+r(k)+1$ and take $\delta_{L(M)}$ to be the boundary of the component of the complement of $\gamma_{r(k)+n(r)+1}$ containing $p_{1}$. 
Finally since $V \cap V_{n}=\emptyset, n>0$, the above results clearly imply that $V$ is bounded.

We are now in a position to prove the main result of this section.

Theorem 3.4. $A$ function $f$ in the class $F$ has no wandering component $U$ of the set $N(f)$ such that $U_{n}$ is multiply-connected for all $n \in \mathbf{N}$.

Proof. The proof is by contradiction. Suppose that $f(z)=z+R(z) e^{Q(z)}$ is a function in $F$ with a wandering domain $U$ such that $U_{n}$ is multiply-connected for all $n \in \mathbf{N}$. We may then take $k \in \mathbf{N}$ and a curve $\gamma$ in $U_{k}=V$ satisfying the conditions of Lemma 3.3. Denoting hyperbolic distance in $W$ by $[\cdot, \cdot]_{W}$ and recalling that $V$ is bounded we see that there exists a constant $L$ such that $\left[z_{1}, z_{2}\right]_{V} \leq L$ for all $z_{1}, z_{2} \in \gamma$. Writing $f^{n}\left(z_{i}\right)=w_{n, i}, i=1,2$, it follows from Schwarz's lemma that $\left[w_{n, 1}, w_{n, 2}\right]_{f^{n}(V)} \leq L$ for all $w_{n, 1}, w_{n, 2} \in \gamma_{n}$. We also know that $V$ is a wandering domain and so, for large $n, f^{n}(V) \subset \Omega$ where $\Omega$ is the plane punctured at the points 0,1 . So, for large $n$, we have

$$
L \geq\left[w_{n, 1}, w_{n, 2}\right]_{f^{n}(V)} \geq\left[w_{n, 1}, w_{n, 2}\right]_{\Omega}
$$

for all $w_{n, 1}, w_{n, 2} \in \gamma_{n}$.

Using the estimates for hyperbolic distance in $\Omega$ given in $[1$, p. 17,18$]$ we see that there exist real constants $M_{0}, \lambda>0$ such that if $w_{n, 1}, w_{n, 2}$ are two points lying on $\gamma_{n}$ such that $\left|w_{n, 1}\right|,\left|w_{n, 2}\right|>M_{0}$ then

$$
L \geq\left[w_{n, 1}, w_{n, 2}\right]_{\Omega} \geq \lambda \int_{\sigma} \frac{|d z|}{|z| \log |z|} \geq \lambda \int_{\left|w_{n, 1}\right|}^{\left|w_{n, 2}\right|} \frac{d|z|}{|z| \log |z|}
$$

where $\sigma$ is a path joining $w_{n, 1}$ to $w_{n, 2}$. Hence for such points

$$
\begin{gathered}
\lambda\left(\log \log \left|w_{n, 2}\right|-\log \log \left|w_{n, 1}\right|\right) \leq L, \\
\Rightarrow \quad \log \log \left|w_{n, 2}\right| \leq \frac{L}{\lambda}+\log \log \left|w_{n, 1}\right|, \\
\Rightarrow \quad \log \left|w_{n, 2}\right| \leq A \log \left|w_{n, 1}\right|, \\
\Rightarrow \quad\left|w_{n, 2}\right| \leq\left|w_{n, 1}\right|^{A}
\end{gathered}
$$

where $A$ is a positive constant dependent only on $\gamma$.

We now take a real number $M>2 M_{0}$ anda curve $\delta_{L(M)}$ which satisfies the conditions of Lemma 3.3. We see that there exist points $w_{L(M), 1}, w_{L(M), 2}$ on $\delta_{L(M)}$ such that $Q\left(w_{L(M), 1}\right)$ is real and positive and $Q\left(w_{L(M), 2}\right)$ is real and negative. Thus, for $M$ sufficiently large,

$$
\begin{aligned}
\left|f\left(w_{L(M), 1}\right)\right| & =\left|w_{L(M), 1}+R\left(w_{L(M), 1}\right) e^{Q\left(w_{L(M), 1}\right)}\right| \\
& >\left|e^{(1 / 2) Q\left(w_{L(M), 1}\right)}\right|>\left|w_{L(M), 1}\right|>M_{0}
\end{aligned}
$$


and

$$
\begin{aligned}
M_{0}<(1 / 2)\left|w_{L(M), 2}\right| & <\left|f\left(w_{L(M), 2}\right)\right|=\left|w_{L(M), 2}+R\left(w_{L(M), 2}\right) e^{Q\left(w_{L(M), 2}\right)}\right| \\
& <(3 / 2)\left|\left(w_{L(M), 2}\right)\right| .
\end{aligned}
$$

It follows that $f\left(w_{L(M), 1}\right), f\left(w_{L(M), 2}\right)$ also satisfy (3.1) and so

$$
\begin{aligned}
\left|e^{(1 / 2) Q\left(w_{L(M), 1}\right)}\right|<\left|f\left(w_{L(M), 1}\right)\right| & \leq\left|f\left(w_{L(M), 2}\right)\right|^{A}<(3 / 2)^{A}\left|w_{L(M), 2}\right|^{A} \\
& \leq(3 / 2)^{A}\left|w_{L(M), 1}\right|^{A^{2}} .
\end{aligned}
$$

From Lemma 3.3 we can take $M$ and hence $\left|w_{L(M), 1}\right|$ to be arbitrarily large which is clearly a contradiction. This completes the proof of Theorem 3.4.

\section{Conjugation with quasiconformal maps}

Finally, we consider conditions (iii) and (iv) of Theorem B. We show that all functions in the class $F$ satisfy these conditions if the word 'entire' is replaced by 'meromorphic' in condition (iii). This is sufficient to allow the use of the proof of Theorem B modified to apply to meromorphic functions. It is clear that for each function $f$ in the class $F$ we must consider families of the form

$G_{K}(f)=\left\{f_{\Phi}=\Phi f \Phi^{-1}: \Phi\right.$ is $K$-quasiconformal fixing $0,1, \infty, f_{\Phi}$ is meromorphic $\}$.

Throughout the following section we will assume that if $f(z)=z+R(z) e^{Q(z)}$, where $R$ is a rational function and $Q$ is a polynomial of degree $q$, then $K q<q+1$. The only result about quasiconformal maps that we use is the following (see, for example, [12] for a proof of this result and for more background material).

Lemma 4.1. If $f$ is a $K$-quasiconformal homeomorphism of the complex plane, fixing 0 and $\infty$ then there is a constant $C$ such that for large $z$ we have

$$
|f(z)|<C|z|^{K} \quad \text { and } \quad\left|f^{-1}(z)\right|<C|z|^{K} .
$$

We also need the following result proved in [8].

Lemma 4.2. Suppose that

$$
f(z)=z+a_{m+1}\left(z-z^{\prime}\right)^{m+1}+\cdots
$$

near $z=z^{\prime}$, where $a_{m+1} \neq 0$ and $m \geq 1$. Then there exist $m$ equally spaced rays at $z^{\prime}$ and $m$ regions $D_{i}, 1 \leq i \leq m$, each of which lies between two adjacent rays and is bounded by a smooth curve which is tangent to the rays at $z^{\prime}$, such that $f\left(D_{i}\right) \subset D_{i}$ and, in $D_{i}, f^{n}(z) \rightarrow z^{\prime}$ in the direction of the bisector, as $n \rightarrow \infty$. The regions $D_{i}$ are known as petals. 
We are now in a position to show that functions in the class $F$ satisfy the modified form of condition (iii).

Lemma 4.3. For a specified $K>1$, and a function $f$ in the class $F$, the family $G_{K}(f)$ can be expressed uniquely in terms of a finite set of complex parameters $X_{1}, \ldots, X_{n(K, f)}$.

Proof. We take a function $f$ in the class $F$ and recall that $f$ can be written in the form

$$
f(z)=z+S(z) e^{Q(z)} / T(z)
$$

where $S, T, Q$ are polynomials. We may assume that $S$ and $T$ have leading coefficients equal to one and have no common roots. We denote the degrees of $S$, $T, Q$ by $s, t, q$ respectively. We write $S(z)=\prod_{i}\left(z-z_{i}\right)^{s(i)}$, where $\sum_{i} s(i)=s$, and note that the fixed points of $f$ are precisely the points $z_{i}$. We now take a function $f_{\Phi}$ from the family $G_{k}(f)$. A point $z$ is a fixed point of $f_{\Phi}$ if and only if $f_{\Phi}(z)=\Phi f \Phi^{-1}(z)=z$ implying $f \Phi^{-1}(z)=\Phi^{-1}(z)$. Thus $\Phi^{-1}(z)=z_{i}$ giving $z=\Phi\left(z_{i}\right)$, for some $z_{i}$.

We now write $T(z)=\prod_{j}\left(z-w_{j}\right)^{t(j)}$, and observe that the poles of $f$ are precisely the points $w_{j}$. Using a similar argument to the above we see that a point $z$ is a pole of $f_{\Phi}$ if and only if $f_{\Phi}(z)=\Phi f \Phi^{-1}(z)=\infty$ implying $f \Phi^{-1}(z)=$ $\Phi^{-1}(\infty)=\infty$. Thus $\Phi^{-1}(z)=w_{j}$ giving $z=\Phi\left(w_{j}\right)$, for some $w_{j}$.

It follows from the above work that

$$
f_{\Phi}(z)=z+S_{\Phi}(z) e^{g(z)} T_{\Phi}(z)
$$

for some polynomials $S_{\Phi}, T_{\Phi}$ and some entire function $g$. We now use Lemma 4.1 to see that the maximum modulus of $f_{\Phi}$ satisfies $M\left(f_{\Phi}, r\right)=O\left(\exp \left(r^{K q}\right)\right)$ and hence $g$ must be a polynomial $Q_{\Phi}$ of degree $q^{\prime} \leq K q<q+1$. We now use the same reasoning to deduce that $Q$ has degree at most $K q^{\prime}$. As $K(q-1)<q$, it follows that $Q_{\Phi}$ must have degree equal to $q$.

It is clear that $T_{\Phi}$ can be written as $T_{\Phi}(z)=\prod_{j}\left[z-\Phi\left(w_{j}\right)\right]^{t(j)}$ and so $T_{\Phi}$ has degree $t$.

We now consider the polynomial $S_{\Phi}$. The roots this has are the points $\Phi\left(z_{i}\right)$ but it is not clear what orders these roots have. Suppose $z_{i}$ is a multiple root of $S$, i.e. $s(i)>1$. Then, near $z_{i}, f$ satisfies the conditions of Lemma 4.2 and we see that there are $s(i)-1$ petals at $z_{i}$. It is easy to see that these are mapped by $\Phi$ onto $s(i)-1$ petals for $f_{\Phi}$ at $\Phi\left(z_{i}\right)$ and so $\Phi\left(z_{i}\right)$ is a root of $S_{\Phi}$ of order $s(i)$. We deduce that $S_{\Phi}$ can be written as $S_{\Phi}(z)=\prod_{i}\left[z-\Phi\left(z_{i}\right)\right]^{s(i)}$ and so $S_{\Phi}$ is of degree $s$.

It follows that the family $G_{K}(f)$ can be expressed in terms of a finite set of parameters $X_{1}, \ldots, X_{n(K, f)}$ as required where $n(K, f)=s+t+q+1$. The parameters are the zeros of $S_{\Phi}$ and $T_{\Phi}$ and the coefficients of $Q_{\Phi}$. 
We now consider the effect of letting $\Phi$ depend on a parameter $t$ and find that the following result holds, and hence functions in the class $F$ satisfy condition (iv).

Lemma 4.4. If, for a given family $G_{K}(f)$, where $f \in F$, each homeomorphism $\Phi=\Phi(t, z)$ depends on a system of parameters $t$ which vary in an open neighbourhood $\mathcal{I}$ of the origin of $\mathbf{R}^{M}$ for some $M$, and $\Phi(0, z)=z$, $\Phi(t, z) \in C^{1}(\mathcal{I})$ for fixed $z$, then for some open subset $\mathcal{I}^{\prime}$ of $\mathcal{I}$, the parameters $X_{i}$ are in $C^{1}\left(\mathcal{I}^{\prime}\right)$.

Proof. From Lemma 4.3 we know that if $f(z)=z+S(z) e^{Q(z)} / T(z)$ where $S, T, Q$ are polynomials of degree $s, t, q$ respectively then each function $f_{\Phi}=$ $\Phi f \Phi^{-1}$ in $G_{K}(f)$ is of the form

$$
f_{\Phi}(z)=z+S_{\Phi}(z) e^{Q_{\Phi}(z)} / T_{\Phi}(z)
$$

where $S_{\Phi}, T_{\Phi}, Q_{\Phi}$ are polynomials of degree $s, t, q$ respectively.

Writing $S(z)=\prod_{i}\left(z-z_{i}\right)^{s(i)}$ we also know that $S_{\Phi}$ can be written as $S_{\Phi}(z)=$ $\prod_{i}\left[z-\Phi\left(z_{i}\right)\right]^{s(i)}$. Similarly, if $T(z)=\prod_{j}\left(z-w_{j}\right)^{t(j)}$, then $T_{\Phi}(z)=\prod_{j}[z-$ $\left.\Phi\left(w_{j}\right)\right]^{t(j)}$. Thus $S_{\Phi}$ and $T_{\Phi}$ are uniquely determined by the functions $\Phi\left(z_{i}\right)$ and $\Phi\left(w_{j}\right)$ all of which are in $C^{1}(\mathcal{I})$.

We now write $Q_{\Phi}(z)=a_{q} z^{q}+\cdots+a_{0}$ where the $a_{k}, 0 \leq k \leq q$, depend only on $t$. The result will clearly be proved if we show that each $a_{k}$ is in $C^{1}\left(\mathcal{I}^{\prime}\right)$ for some open subset $\mathcal{I}^{\prime}$ of $\mathcal{I}$.

As shown in the proof of Theorem 2.1, $f$ has infinitely many critical points $\alpha_{c}, c \in \mathbf{N}$. The critical points of $f_{\Phi}$ are precisely $\alpha_{c}(t)=\Phi\left(t, \alpha_{c}\right)$. Each such $\alpha_{c}(t)$ satisfies

$$
\begin{aligned}
F_{c}\left[\Phi\left(t, z_{i}\right), \Phi\left(t, w_{j}\right), a_{k}(t), t\right]=T_{\Phi}^{2}\left(\alpha_{c}\right) \frac{\partial}{\partial z} f_{\Phi}\left(\alpha_{c}(t)\right) \\
=T_{\Phi}^{2}\left(\alpha_{c}(t)\right)+\left[-S_{\Phi}\left(\alpha_{c}(t)\right) T_{\Phi}^{\prime}\left(\alpha_{c}(t)\right)+S_{\Phi}^{\prime}\left(\alpha_{c}(t)\right) T_{\Phi}\left(\alpha_{c}(t)\right)\right] e^{Q_{\Phi}\left(\alpha_{c}(t)\right)} \\
\quad+S_{\Phi}\left(\alpha_{c}(t)\right) T_{\Phi}\left(\alpha_{c}(t)\right) Q_{\Phi}^{\prime}\left(\alpha_{c}(t)\right) e^{Q_{\Phi}\left(\alpha_{c}(t)\right)}=0 .
\end{aligned}
$$

We see that each $F_{c}$ depends analytically on each of the parameters of $f_{\Phi}$ and belongs to $C^{1}(\mathcal{I})$.

We have $\alpha_{c}(0)=\alpha_{c}$ and so, at $t=0$,

$$
\begin{aligned}
\frac{\partial F_{c}}{\partial a_{k}}= & {\left[-S\left(\alpha_{c}\right) T^{\prime}\left(\alpha_{c}\right)+S^{\prime}\left(\alpha_{c}\right) T\left(\alpha_{c}\right)+S\left(\alpha_{c}\right) T\left(\alpha_{c}\right) Q^{\prime}\left(\alpha_{c}\right)\right] \alpha_{c}^{k} e^{Q\left(\alpha_{c}\right)} } \\
& +k S\left(\alpha_{c}\right) T\left(\alpha_{c}\right) \alpha_{c}^{k-1} e^{Q\left(\alpha_{c}\right)} .
\end{aligned}
$$

We claim that there are $q+1$ choices of $c$ for which the vectors

$$
\underline{u}_{c}=\left(\frac{\partial F_{c}}{\partial a_{0}}, \ldots, \frac{\partial F_{c}}{\partial a_{q}}\right)
$$


are linearly independent at $t=0$. If not, then all of the $\underline{u}_{c}$ span a space in $\mathbf{C}^{q+1}$ of dimension at most $q$. This implies that there exists a vector $\underline{v}=\left(v_{0}, \ldots, v_{q}\right)$ such that $\underline{v} \not \equiv \underline{0}$, and for each $c$ at $t=0$

$$
v_{0} \frac{\partial F_{c}}{\partial a_{0}}+\cdots+v_{q} \frac{\partial F_{c}}{\partial a_{q}}=0 .
$$

Combining (4.1) and (4.2) we see that the polynomial $P$ where

$$
P(z)=\sum_{k=0}^{q} v_{k}\left\{\left[-S(z) T^{\prime}(z)+S^{\prime}(z) T(z)+S(z) T(z) Q^{\prime}(z)\right] z^{k}+k S(z) T(z) z^{k-1}\right\}
$$

then vanishes for each $\alpha_{c}$ and hence for all $z$. The highest power of $z$ in this polynomial is $s+t+2 q-1$ and this comes from the term $v_{k} S(z) T(z) Q^{\prime}(z) z^{q}$ and so $v_{q}=0$. Using the same line of argument it follows successively that $v_{q-1}=0$, $v_{q-2}=0, \ldots, v_{0}=0$ and so $\underline{v} \equiv \underline{0}$ which is a contradiction.

We are now able to take $q+1$ choices of $c$, which we label as $c(d)$ where $d=0,1, \ldots, q$, such that the vectors $\underline{u}_{c(d)}$ are linearly independent at $t=0$. It follows that the matrix

$$
\left(\frac{\partial F_{c(d)}}{\partial a_{k}}\right), \quad 0 \leq d, k \leq q,
$$

is non-singular at $t=0$. Letting $\underline{b}(t)$ be the vector with components $\Phi\left(t, z_{1}\right)$, $\Phi\left(t, z_{j}\right)$, and $\underline{a}(t)=\left(a_{0}(t), \ldots, a_{q}(t)\right)$ it now follows from the implicit function theorem that there is a $C^{1}$ function $g: \underline{b}(t) \rightarrow \underline{a}(t)$ in a neighbourhood of $\underline{a}(0)$. We know that all the components of $\underline{b}$ are in $C^{1}(\mathcal{I})$ and hence all the $a_{k}, 0 \leq k \leq q$, are in $C^{1}\left(\mathcal{I}^{\prime}\right)$ for some open subset $\mathcal{I}^{\prime}$ of $\mathcal{I}$ as required.

Using the results of the last three sections we are now able to prove Theorem $\mathrm{C}$ using essentially the same argument as that used to prove Theorem B in $[2$, Section 7].

The author wishes to thank Professor I.N. Baker for his helpful suggestions and the SERC for their financial support.

\section{References}

[1] Ahlfors, L.: Conformal invariants. - McGraw-Hill, New York, 1973.

[2] BAKER, I.N.: Wandering domains in the iteration of entire functions. - Proc. London Math. Soc. (3) 49, 1984, 563-576.

[3] BAKER, I.N., J. KotUS, and LÜ YINIAN: Iterates of meromorphic functions I. - Ergodic Theory Dynamical Systems 11, 1991, 241-248.

[4] BAKER, I.N., J. KotUS, and LÜ YINIAN: Iterates of meromorphic functions III. - Ergod. Theory Dynam. Systems (submitted).

[5] BAKER, I.N., J. Kotus, and Lü Yinian: Iterates of meromorphic functions IV. - Manuscript. 
[6] BERS, L.: On Sullivan's proof of the finiteness theorem and the eventual periodicity theorem. - Amer. J. of Math. 109, 1987, 833-852.

[7] Eremenko, A., and M.Yu. Lyubich: Iterates of entire functions. - Preprint 6 of the Physico-technical Institute of Low Temperatures, Ukranian S.S.R. Academy of Sciences, Kharkov, 1984 (in Russian).

[8] Fatou, P.: Sur les équations fonctionelles. - Bull. Soc. Math. France 47, 1919, 161-271; 48, 1920, 33-94, 208-314.

[9] Fatou, P.: Sur l'itération des fonctions transcendantes entières. - Acta Math. 47, 1926, 337-370.

[10] Goldberg, L.R., and L. KeEn: A finiteness theorem for entire functions. - Ergod. Theory Dynam. Systems 6, 1986, 183-192.

[11] Hayman, W.K.: Meromorphic functions. - The Clarendon Press, Oxford, 1964.

[12] Lehto, O., and K.I. Virtanen: Quasiconformal mappings in the plane. - SpringerVerlag, 1973.

[13] Sullivan, D.: Itération des fonctions analytiques complexes. - C. R. Acad. Sci. Paris Sér. A-B 294, 1982, 301-303.

[14] Sullivan, D.: Quasiconformal homeomorphisms and dynamics I. Solution of the FatouJulia problem on wandering domains. - Ann. of Math. 122, 1985, 401-418.

Imperial College of Science, Technology and Medicine

Department of Mathematics

London SW7 2AZ

England

Received 14 June 1990 